40-1| 2015

Enjeux contemporains en études irlandaises - In

Memoriam Paul Brennan

\title{
Clusters et networks en Irlande : un modèle de développement économique?
}

Valérie Peyronel

\section{(2) OpenEdition \\ Journals}

Édition électronique

URL : http://journals.openedition.org/etudesirlandaises/4546

DOI : 10.4000 /etudesirlandaises. 4546

ISSN : 2259-8863

\section{Éditeur}

Presses universitaires de Rennes

Édition imprimée

Date de publication : 30 juin 2015

Pagination : 105-117

ISBN : 978-2-7535-4082-8

ISSN : 0183-973X

Référence électronique

Valérie Peyronel, «Clusters et networks en Irlande : un modèle de développement économique? », Études irlandaises [En ligne], 40-1 | 2015, mis en ligne le 30 juin 2017, consulté le 19 avril 2019. URL http://journals.openedition.org/etudesirlandaises/4546; DOI : 10.4000/etudesirlandaises.4546 


\title{
Clusters et networks en Irlande : un modèle de développement économique?
}

\author{
Valérie Peyronel \\ Université Sorbonne Nouvelle - Paris 3
}

\section{Résumé}

Dans une économie de plus en plus globalisée, l'importance de liens et de relations plus étroits entre les différents acteurs économiques est apparue de plus en plus clairement. Les regroupements et les alliances sont devenus les outils indispensables de la compétitivité tant pour les entreprises que pour les institutions traditionnellement supposées relever de la sphère publique, comme l'enseignement et la recherche, désormais forcées, en raison de déficits publics croissants, de trouver d'autres sources de revenus et de se lancer dans la compétition. C'est pourquoi se sont formés des grappes (clusters) et des réseaux (networks) de diverses natures, caractérisés par la concentration des moyens et la complémentarité, à l'échelle internationale, nationale ou régionale, impliquant des acteurs publics ou privés, ou des partenariats public-privé. Le Tigre celtique n’a pas été épargné par ce phénomène économique, et le développement économique remarquable de l'Irlande s'est appuyé en partie sur l'éclosion de ces noyaux et réseaux dans divers secteurs de la vie économique. Cependant, l'émergence et la multiplication de ces structures pose nombre de questions sur leur mise en œuvre et leurs effets, et sur le fossé éventuel entre théorie et pratique.

Mots clés : État irlandais (République d'Irlande), Irlande - politiques économiques et sociales, industrie

\section{Abstract}

In an increasingly globalised economy, the importance of tighter links and relationships between the various economic stakeholders has become more and more obvious. Connections and alliances have become the indispensable instruments of competitiveness both for enterprises and for institutions traditionally related to the public sector, like the higher education and research institutions which, as a result of constantly inflating public deficits, have had to find new sources of funding and become competitive too. This is the reason why a large variety of regional, national and international clusters and networks have taken shape, all characterised by the concentration of means and by complementarity, involving public or private stakeholders or public-private partnerships. The Celtic Tiger has not been spared by this economic phenomenon and the remarkable economic development of Ireland has partly depended on the development of such clusters and networks in various economic sectors. However, the emergence and the multiplication of these structures call the issues of their implementation and effects and of the possible gap between theory and practice in question.

Keywords: Irish State (Republic of Ireland), Ireland - socio-economic policies, industries 
Dans le contexte complexe de globalisation et de course à la compétitivité, les relations entre les différents agents économiques, publics comme privés, et plus particulièrement entre les entreprises, d'une part, et entre ces dernières et leur environnement économique, d'autre part, sont devenues des outils économiques et managériaux indispensables. De même, la géographie de l'économie a changé, tandis que se dessinent des noyaux et des réseaux relationnels. Dans ce contexte, le phénomène des clusters (que l'on peut traduire par grappes, groupes ou essaims) et des networks (réseaux) a pris une ampleur notoire, notamment au niveau européen. En décembre 2006, le Conseil européen pour la compétitivité (European Competitiveness Council) a désigné les clusters comme l'une des neuf priorités destinées à renforcer l'innovation en Europe.

L'Irlande, dont le développement de regroupements de ce type a été relativement tardif par rapport à ses voisins européens, et particulièrement par rapport au Royaume-Uni, a néanmoins vu le phénomène s’amplifier dans les années 1980 et 1990 , mais cette réalité a fait l'objet de controverses. Dans un rapport publié par Forfás en $2007^{1}$, Eleanor Doyle et Connell Flanning soulignaient que plusieurs des sept études sur les clusters en Irlande publiées entre 1994 et 2002 et qu'elles avaient passées en revue ${ }^{2}$ concluaient qu'il n'était pas nécessaire de se focaliser sur les clusters, puisque l'économie irlandaise s'était déjà relativement bien développée avant leur apparition. Certaines études suggéraient même de trouver des modèles autres ${ }^{3}$. Les analyses d'O'Gorman, O’Malley et Mooney de $1997^{4}$ portant sur l'industrie des logiciels, celle de Gallagher, Doyle et O'Leary datant de $2002^{5}$ et, plus récemment, une étude du Department of Enterprise, Trade and Employment de $2008^{6}$, en s'intéressant à la question de la compétitivité de l'économie irlandaise, mettaient également en évidence que le phénomène de "clusterisation " avait été essentiellement circonscrit aux domaines du logiciel, de l'électronique et du matériel de télécommunications.

Après avoir défini les clusters et les networks en tant que modèles et examiné les raisons de l'intérêt qui leur est porté, nous nous interrogerons sur leur mise en œuvre en Irlande, puis analyserons les limites de l'application des modèles.

1. Eleanor Doyle et Connell Fanning, «The Role for Clusters in Irish Economic Development Policy ", Perspectives on Irish Productivity, Dublin, Forfás, March 21, 2007, chapter 16 [http://www.forfas.ielpublications/search.jsp?ft=/ publications/2007/Title, 731,en.php], page consultée le 31 mai 2014.

2. Ibid, p. 276.

3. Ibid.

4. Colm O'Gorman, Eoin 0'Malley et John Mooney, The Irish indigenous software industry, An application of Porter's cluster analysis, Dublin, National Economic and Social Council, 1997.

5. Liam Gallagher, E. Doyle and Eoin O’Leary, "Creating the Celtic Tiger and sustaining Economic Growth: A Business Perspective ", Quarterly Economic Commentary, Avril 2002.

6. Department of Enterprise, Trade and Development, Knowledge and Enterprise Clusters in Ireland: an overview, 2008. 


\section{Les clusters et les networks au cœur des modèles de développement économique anglo-saxons}

L'intérêt majeur pour le phénomène des clusters et des networks (réseaux) remonte à la fin des années 1980, probablement en raison de l'essor économique qui se dessinait à l'approche des Golden 90s, décennie de dynamisme et de croissance, mais aussi de remaniements, de fusions et de concurrence accrue.

Michael Porter ${ }^{7}$ a consacré nombre de ses travaux à l'étude de ce type de structures, en commençant par son ouvrage Competitive Advantage of Nations (1990) ${ }^{8}$. Porter est parti du premier postulat que le succès rencontré par certaines nations industrielles tenait à leur capacité à établir des systèmes de liaison verticale (par exemple : vendeurs/acheteurs) et/ou horizontale (partage de fournisseurs, de clients, de technologie, de circuits de distribution) ${ }^{9}$. Il a ensuite progressé vers la définition des clusters (noyaux), que nous analyserons ici en contraste avec les networks (réseaux).

Ainsi les clusters peuvent-ils se définir comme des " concentrations géographiques d'entreprises et d'institutions interconnectées dans un domaine particulier ${ }^{10}$ ", de fournisseurs spécialisés, de fournisseurs de services, d'entreprises de services associés, et d'institutions associées (comme des universités, ou des associations professionnelles, par exemple) qui peuvent être en concurrence, mais se caractérisent également par de forts liens de coopération.

La particularité de la zone géographique où se situe le cluster, outre d'être définie par ses limites physiques (ce en quoi Porter est d'ailleurs rejoint par d'autres auteurs, comme Michael Enright ${ }^{11}$, par exemple), est de représenter une entité économique conceptuelle : un marché de l'emploi, une zone métropolitaine, un bassin d'échanges industriels ou commerciaux. Mais il s'y ajoute aussi, selon Michael Porter, une notion d'avantage concurrentiel, qui peut même constituer la base de la formation du cluster : «les clusters incluent une batterie d'industries et d'autres entités reliées entre elles et importantes pour la compétitivité ${ }^{12}$.

7. Michael E. Porter, né en 1947, professeur de stratégie d'entreprise à l’université d'Harvard (Cambridge, Massachussetts).

8. Michael E. Porter, Competitive Advantage of Nations, New York, The Free Press, 1990.

9. Ibid., p. 149.

10. Michael E. Porter, "Clusters and the New Economics of Competition ", Harvard Business Review, NovemberDecember 2008, p. 78: "Geographic concentrations of interconnected companies and institutions in a particular field".

11. Michael J. Enright, "The Geographic Scope of Competitive Advantage ", dans Elke Dirven, Joost Groenewegen and Sjef Van Hoof, Stuck in the Region? Changing Scales for Regional Identity, Utrecht, Netherlands Geographic Studies, 155, 1993, p. 87-102.

12. Michael. E. Porter, "Clusters and the New Economics of Competition », op. cit., p. 78 : «Clusters encompass an array of linked industries and other entities important to competition ". 
La proximité géographique au sein du cluster constitue d'ailleurs l'un des éléments non négligeables de sa compétitivité et de ses éléments constitutifs (entreprises, institutions, associations) car cette proximité est génératrice, d'une part, de synergies, elles-mêmes sources d'économie de temps et d'énergie et, d'autre part, d'innovation, comme le rappelait un rapport établi pour la Commission Européenne en $2006^{13}$. Quel que soit le degré de performance des concurrents hors cluster, ils ne peuvent égaler les gains de productivité ainsi réalisés, qui représentent un avantage comparatif (a competitive edge) notoire pour les membres du cluster. L'appartenance à un cluster n'interdit cependant pas la concurrence interne. Par ailleurs, les alliances au sein du cluster sont multiformes et n'incluent pas nécessairement tous ses membres à la fois. C’est la dynamique des interconnections qui rend l'ensemble plus compétitif (peut-être justement parce que la concurrence interne est possible).

Les clusters répondent donc au triple objectif suivant :

- économies d'échelle,

- complémentarité et compétitivité,

- mais aussi développement régional.

Les networks, pour leur part, peuvent se définir comme des relations de coopération établies de manière plus ou moins formelle entre des entreprises et/ou des institutions. Ils sont généralement perçus comme le résultat d'un changement dans la donne concurrentielle au sein d'une économie de plus en plus globalisée. Ainsi Alan M. Rugman et Joseph D’Cruz postulent-ils que :

la stratégie d'entreprise s'est traditionnellement focalisée sur la rivalité et les structures d'entreprises sur la hiérarchie. Cette approche cède maintenant le pas à l'idée qu'il est préférable de fonder une compétitivité internationale durable sur des relations de coopération au sein d'une structure de réseau ${ }^{14}$.

S'appuyant sur la métaphore de la forêt tropicale, qu'ils opposent à celle de la jungle du monde des affaires où indépendance et rivalité prévalent, Håkan Håkansson et ses co-auteurs soulignent l'interaction qui prévaut dans la structure en réseaux :

13. The High Level Advisory Group on Clusters, The European Cluster Memorandum: Promoting European Integration through Clusters, Europe INNOVA Initiative of the European Commission, January 2006, [www.kkt.lt/ get.php?f.9], page consultée le 28 juin 2014.

14. Alan M. Rugman et Joseph R. D’Cruz, Multinationals as Flagship Firms - Regional Business Networks, Oxford, Oxford University Press, 2000, p. 1: «Business strategy traditionally has focused on rivalry and business structure on hierarchy. Such thinking is now giving way to the idea that sustainable international competitiveness can best be achieved through co-operative relationships in a business network structure. " 
La métaphore de la forêt tropicale humide met l'accent sur l'importance de l'interaction avec les autres. La vie d'une entreprise dépend des autres et vice-versa. Les entreprises évoluent en relation les unes avec les autres dans le temps, selon des processus producteurs d'une grande diversité. Ces processus sont bénéfiques tant aux entreprises individuelles qu'au paysage dans son ensemble. Par conséquent l'hypothèse qui soustend les modèles économiques rattachés à la métaphore de la forêt tropicale humide veut que l'interaction multidimensionnelle soit une caractéristique fondamentale du paysage de l'entreprise ${ }^{15}$.

Les réseaux peuvent être classés en deux catégories : les hard networks et les soft networks. Les premiers comprennent des entreprises qui se regroupent pour produire, distribuer, acheter de manière conjointe ou collaborer pour le développement de produits et de marchés communs. Le risque commercial est partagé :

Les réseaux durs sont plus formels, requièrent généralement un engagement contractuel en matière de partage et d'échange de ressources et de profits, et sont par conséquent plus restrictifs en termes d'adhésion et forment des associations très structurées et formalisées ${ }^{16}$.

Les seconds visent à développer les liens qui permettent aux entreprises de résoudre des difficultés communes, de partager de l'information, de participer ensemble au développement de la formation et des compétences.

Les réseaux mous sont des groupes relativement informels, au sein desquels l'adhésion est plus ou moins ouverte, et qui impliquent une coopération pour traiter des problèmes communs, partager de l'information ou atteindre des résultats pour le bénéfice mutuel des membres du réseau ${ }^{17}$.

15. Håkan Håkansson, David Ford, Lars-Erik Gadde, Ivan Snehota, Alexandra Waluszewski, Business in Networks, Chichester, John Wiley \& Sons, 2009, p. 6: "The rainforest metaphor emphasizes the importance of interaction with others. The life of one company is dependent on others and vice-versa. Companies evolve in relation to each other over time in processes that produce great variety. These processes are beneficial for both individual companies and the landscape as a whole. Hence the assumption that underlies those economic frameworks that relate to the rainforest metaphor is that multidimensional interaction is a basic feature of the business landscape. "

16. Kirk J.Ring, Ana Maria Pereda et James J. Chrisman, "Business Networks and Economic Development in Rural Communities in the United Sates ", Entrepreneurship, Theory and Practice, January 1 ${ }^{\text {st }}, 2010$, p 7, [http:// www.allbusiness.com/government/government-bodies-offices-us-federal-government/13838663-1.html], page consultée le 10 février 2014: "Hard networks are more formal, generally require some form of contractual commitment with regard to the sharing and exchange of resources and profits and are therefore more restrictive in terms of membership, and highly structured and formalized associations."

17. Ibid.: "Soft networks are relatively informal groups with more or less open membership that involve cooperation to deal with common problems, share information or achieve outcomes that are mutually advantageous to network members." 
Contrairement aux clusters, la notion de concentration géographique n'est pas indispensable dans le concept du network, bien qu'elle ne soit pas exclue dans les faits.

\section{圈 L'émergence des concepts de clusters et de networks en Irlande}

Les clusters et les networks se sont développés dans l'économie irlandaise, les premiers étant cependant, en raison de leur concentration géographique, plus aisés à identifier que les seconds.

\section{Les clusters}

Le rapport Culliton ${ }^{18}$, publié en 1992, fut le premier document de politique économique recommandant, dans le cadre d'une réorganisation du tissu industriel irlandais, et en particulier en vue d'une amélioration de la productivité et de la compétitivité de l'industrie irlandaise, la mise en œuvre d'une politique " sélective " pour établir des clusters industriels autour de " sources d'avantage concurrentiel national " (sources of national competitive advantage). Il s'agissait donc d'identifier les points forts de l'économie irlandaise, c'est-à-dire les secteurs les plus attractifs, reconnaissables par le fait qu'ils étaient particulièrement ciblés par les investissements étrangers.

Ash Amin et John Tomaney, co-éditeurs de Behind the Myth of European Union : Prospects for Cohesion ${ }^{19}$ publié en 1995, ont analysé qu'en effet, les clusters ont été envisagés dans un premier temps en Irlande comme un moyen de réorienter le soutien public aux entreprises, jusqu'alors essentiellement dirigé vers l'investissement direct étranger (Foreign Direct Investment), vers les entreprises irlandaises : "La promotion de forces locales pour créer des noyaux industriels d'entreprises connexes et qui se renforcent mutuellement ${ }^{20}$. "

L'intérêt porté à la création de ces nouvelles synergies, capables de propulser l'Irlande au rang des détenteurs de ce que l'on viendrait plus tard à nommer des pôles d'excellence, se doublait de l'insistance croissante, dans le cadre de la distribution des fonds européens, sur le développement et la compétitivité des régions. Or, par l'importance de sa dimension régionale, le cluster correspondait parfaitement à ces attentes européennes.

18. Industrial Policy Review Group, A Time for Change: Industrial Policy for the 1990s, 1992. Ce rapport, rédigé par l'Industrial Policy Review Group preside par Jim Culliton, fut présenté au Minister of Trade and Industry.

19. Ash Amin et John Tomaney, Behind the Myth of European Union: Prospects for Cohesion, London, Routledge, 1995.

20. Ibid. p. 310: "The promotion of local strengths to create industrial clusters of related and reinforcing industries. " 


\section{Les networks}

En 2004, un rapport intitulé Ahead of the Curve, rédigé par l'Economic Strategy Group (un comité mis en place par le gouvernement irlandais pour étudier les moyens à mettre en place pour favoriser la croissance et l'emploi en Irlande) appelait à une plus grande coopération entre les entreprises, les institutions académiques (les universités et écoles) et l'État et concluait :

Des réseaux forts, dirigés par des entreprises, sont nécessaires pour mettre en œuvre le programme stratégique dans leurs domaines d'activité. De plus en plus, ces réseaux faciliteront le transfert des connaissances, dissémineront la connaissance des marchés, encourageront l'innovation, informeront les programmes de recherche et identifieront les besoins en infrastructure spécifiques au développement sectoriel ${ }^{21}$.

L'étude Business Networks on the Island of Ireland, commanditée par Intertrade Ireland et publiée en $2005^{22}$, identifiait essentiellement trois types de réseaux :

- les Business networks, les plus nombreux, se définissant comme un groupe d'entreprises qui décident de collaborer, et pour lesquelles cette collaboration a un impact tangible et chiffrable sur leur activité (soit un gain de compétitivité, soit des gains financiers). Il peut s'agir de distribution conjointe de produits et services, de réseaux de recherche, de formation et de développement managérial, de distribution aux clients, ou de co-production.

- Les Development networks, c'est-à-dire des réseaux informels d'entreprises qui permettent l'échange d'informations ou de services, mais sans qu'en soient attendus des gains de compétitivité ou financiers tels que ceux proposés dans le schéma précédent : les incubateurs ou des réseaux de femmes d'affaires sont des exemples.

- Les Business Support Networks, enfin, qui sont des associations destinées, de manière informelle, à soutenir le développement des entreprises : réseaux entre des associations professionnelles et des collectivités locales, par exemple.

Il convient de noter que les business networks ne reposent pas seulement sur des réseaux d'affaires, mais également sur des relations personnelles et sociales. Cependant, dans le cadre de l'étude, ils ont été définis comme : des groupements d'entreprises directement concernées par la mise en ouvre d'une certaine forme

21. Enterprise Strategy Group, Ahead of the curve: Executive Summary, Forfás, 2004, p xIII, [http://www.forfas.iel medialesg040707_entreprise_strategy_executive_summary.pdff, page consultée le 10 juin 2014: "Strong enterprise-led networks are required that will establish the strategies agenda for their areas of activity. These networks will increasingly facilitate knowledge transfer, disseminate market knowledge, foster innovation, inform the research agenda and identify infrastructure needs specific to sectoral development. "

22. Maire Hunt (Skillnets Ltd), Gerald Doyle (Creative Change Ltd), Dermott McDermott (Momentum) et Paul McCormack (Newry and Mourne Enterprise Agency), Business Networks on the Island of Ireland, Dublin, Intertrade, 2005, p. 15, [www.intertradeireland.com/module.cfm/opt/29/area/Publications/.../323], page consultée le 20 septembre 2014 . 
de commerce et d'affaires. Le réseau peut aussi inclure des associations commerciales, des chambres de commerce, des organismes professionnels ou des agences du secteur public.

\section{Du modèle à la réalisation}

Le rapport Business Networks on the Island of Ireland de 2005 précédemment évoqué, commandité par Intertrade Ireland, identifiait en Irlande 110 clusters et networks (dont 74 Business networks, 18 Development networks et 18 Regional business clusters), regroupant au total 9860 entreprises ou institutions, les grandes entreprises ne représentant que $7 \%$ d'entre elles. Dans la plupart des cas, il s'agissait de réseaux et de clusters récents ( 5 ans et moins ${ }^{23}$ ce qui, dans le cas des clusters, et si l'on compare ces résultats à ceux des études citées par Amin et Tomaney concernant la période 1994-2002, corroborerait l'hypothèse d'une clusterisation récente de l'économie irlandaise.

L'étude de 2005 soulignait également que le potentiel de croissance était particulièrement important pour les business networks, et quatre fois moindre pour les clusters. Ceci est en partie lié à la nature même des deux modèles : le cluster, de par sa concentration géographique, est en effet d'emblée plus concentré24. En termes de taille, les petites entreprises (moins de 50 employés) étaient très largement majoritaires (de $83 \%$ à $93 \%)^{25}$, ce qui reflète l'attractivité des modèles de clusters et de networks pour des entreprises de petite taille qui y recherchent les synergies et les économies d'échelle qui leur manquent : une situation d'autant plus évidente dans une économie irlandaise qui repose largement sur les petites et moyennes entreprises. Par ailleurs, l'étude montrait que, d'une part, plus le réseau était informel, plus la proportion de petites entreprises était élevée, et que, d'autre part, la proportion d'entreprises de taille moyenne était la plus élevée dans les clusters (soit $14 \%$ d'entreprises entre 51 et 250 employés) ${ }^{26}$. La première constatation s'explique par la facilité, pour des petites entreprises, d'intégrer les réseaux informels. La seconde tient à la structure même du cluster souvent constitué autour d'un noyau d'entreprises plus importantes (voire même d'une seule grande entreprise) qui fédère l'essaim. Dans le cas irlandais, ce sont souvent les filiales d'entreprises multinationales qui ont joué ce rôle, ce qui corrobore les recommandations qui figuraient dans le rapport Culliton. En termes de secteurs d'activité, enfin, l'étude montrait, tous modèles confondus, la prédominance des clusters et des

23. Ibid., p. 5. Il est par ailleurs précisé sur cette même page, que l'enquête avait, de surcroit, identifié 17 Business Support Networks, mais que ces derniers n'avaient pas été pris en compte dans l'analyse.

24. Ibid., p. 21.

25. Ibid.

26. Ibid. 
networks multisectoriels (en général propre aux networks) soit 40/110 et l'importance également, des secteurs clé que sont les technologies de l'information (ICT), soit 16 des 110 entités identifiées, et l'industrie alimentaire (food), soit 10/110, des concentrations mono-sectorielles plus propres aux clusters $^{27}$. En effet, le secteur des Technologies de l'Information et de la Communication concentré autour de Dublin, Shannon, Dundalk a été un moteur particulièrement performant de la « clusterisation» en Irlande. L'industrie des logiciels, en particulier, a donné naissance à d'importants clusters, le plus important se situant dans la région de Dublin, d'autres, plus modestes, étant répartis entre Cork, Shannon/Limerick et Galway $^{28}$.

Une étude publiée par le Department of Enterprise, Trade and Employment en $2008^{29}$ a bien mis en évidence le développement des clusters dans trois domaines principaux, relevant tous de technologies avancées : la bio-pharmacie, les techniques d'information et de communication, et les services commercialisés sur les marchés internationaux (internationally traded services). La bio-pharmacie s'est développée autour de Cork, ainsi que dans le Mid West et le South West, tandis qu'un cluster dans un domaine proche, les services médicaux, s'est formé dans la région de Galway. Plusieurs clusters ont pris de l'ampleur autour de Limerick, Cork et Dublin. Dublin demeure également la ville d'accueil d'un International Financial Services Center très bien positionné au niveau international. Lors de l'inauguration du bureau de Waterford de BISYS Hedge Funds Services en 2005, Micheál Martin, Ministre de l'Entreprise, du Commerce et de l'Emploi, avait ainsi déclaré :

Un noyau d'entreprises dans le domaine des services financiers internationaux commence à prendre forme dans le Sud Est. [...] Avec des entreprises financières à Wexford, Kilkenny et Waterford, la région du Sud Est en train d'émerger rapidement comme l'une des localisations préférées de l'industrie des services financiers en dehors de Dublin ${ }^{30}$.

27. Ibid, p. 22.

28. Roy Green, Irish ICT Cluster, OECD Cluster Focus Group Workshop, Utrecht, May 8-9, 2000, [http://www. oecd.org/dataoecd/8/60/2754426.pdf, page consultée le 30 septembre 2014.

29. Department of Enterprise, Trade and Employment, Knowledge and Enterprise Clusters in Ireland, October 2008, [http://www.entemp.ie/tradeleuaffairs/knowledgeandenterpriseclusters.pdf], page consultée le 15 mai 2014.

30. IDA Ireland, Dublin, International Financial Services in Ireland Report, Winter 2005, p. 2 : «A cluster of companies in the international financial services area is beginning to take shape in the South East [...] With financial companies in Wexford, Kilkenny and Waterford, the South East region is quickly emerging as one of the preferred locations for the financial services industry outside Dublin." " 


\section{De la pratique à la théorie : réalités et limites}

La réussite de certains clusters irlandais tend ainsi à démontrer le bien fondé des théories de Porter.

Dans une étude publiée en $2008^{31}$ sur la " clusterisation " des shared services en Irlande, les auteurs rappelaient que ces « services partagés permettent aux entreprises de mutualiser leurs services de gestion (back office procession order) pour parvenir à une plus grande efficacité et offrir des services de meilleure qualité " et démontraient l'adéquation du " diamant " de Porter au développement des shared services en Irlande.

Selon Porter, le développement d'un cluster est en effet fondé sur l'association de quatre facteurs, considérés comme les facettes complémentaires d'un même "diamant ${ }^{32} »$ :

- les facteurs (factor conditions);

- la demande (demand conditions);

- la stratégie, les structures et la rivalité entre les entreprises (firm strategy, structure and rivalry);

- des entreprises rattachées en soutien (supporting and related industries).

De surcroît, les " éventualités " (contingency) et le " gouvernement " (govern$m e n t$, tout en ne faisant pas partie des éléments clé inclus dans le diamant de Porter, contribuent à créer l'environnement économique favorable au développement des clusters.

Dans le cas des shared services, l'étude de 2005 montrait la réunion des éléments nécessaires :

- en termes de facteurs : une main-d'œuvre jeune et surqualifiée ;

- en termes de demande : des entreprises américaines désireuses de consolider leurs services administratifs en un seul endroit au niveau européen ;

- en termes de stratégie, le souci de ces mêmes entreprises de rationaliser leurs opérations pour être plus compétitives.

Enfin les opportunités offertes par le marché européen et le soutien du gouvernement par le biais de l'IDA (Industrial Development Agency) en matière d'IDE (Investissement Direct Etranger) illustraient les facteurs " chance " et " gouvernement ». Cependant, la mise en relation avec les industries locales (supporting and related industries) semblait un point faible, les auteurs de l'étude rejoignant ainsi

31. Competitiveness, European Cluster Mapping Project «Identification, analysis and monitoring of business clusters in Europe ", 22 January 2008, p. 6, [http://www.clusterobservatory.eu/library/100.111.pdf], page consultée le 30 juin 2014: "Shared services centres allow companies to combine back office procession order to achieve increased efficiency and higher quality services."

32. Michael. E. Porter, Competitive Advantage of Nations, op. cit., p. 72. 
nombre de critiques adressées aux clusters irlandais fondés sur de l'investissement étranger qui déplorent le manque d'impact (spillover effects) sur l'industrie locale.

Car en dépit d'exemples réussis, les critiques et le scepticisme, concentrés plutôt sur les clusters que sur les networks plus flexibles, ont été nombreux. La question des retombées des clusters, en particulier lorsque ceux-ci sont fondés sur le déploiement d'activités d'entreprises étrangères implantées en Irlande, est en effet l'une des principales critiques adressées à la "clusterisation ». Pourtant cette opinion doit être nuancée selon les secteurs, si l'on se réfère aux conclusions présentées par le Department of Enterprise, Trade and Employment en 2008 sur le secteur des Technologies de l'Information et de la Communication : "L'une des principales retombées de la présence des principaux fabricants primaires d'équipement (Original Equipment Manufacturers-OEMs) en Irlande est l'existence d'une industrie des logiciels très fructueuse ${ }^{33}$. "Une analyse publiée par South West Regional Authority en juin $2009^{34}$ aboutit à des conclusions similaires. Cette étude démontre que l'activité entrepreneuriale est articulée, dans toutes les zones de clusters, avec des activités d'enseignement supérieur et de recherche (les universités de Cork, de Galway, de Dublin) et des activités de service directement liées aux opérations menées au sein des clusters : recrutement, approvisionnement et construction.

Les rôles respectifs des pouvoirs publics et des entreprises privées, en particulier dans le cas des clusters, ont également été sujets à caution. La création et le fonctionnement des clusters et des networks sont en effet largement dépendants de ce qui était apparu pour la première fois sous le terme de facilitator dans le Danish Network Programme de $1993^{35}$ : un intermédiaire qui puisse identifier les opportunités, mettre les participants potentiels en contact, apporter l'information et éventuellement, l'aide technique nécessaire au projet. Cette aide peut se situer au niveau régional (comme ce fut le cas, par exemple, des Regional Development Agencies en Grande-Bretagne avant leur suppression en 2012), mais peut aussi revêtir la forme d'organismes dédiés : en République d'Irlande, c'est l'une des tâches assignées à Enterprise Ireland, tandis qu'au Nord, c'est Invest NI qui joue principalement ce rôle.

Or, dans le cadre de l'étude de 2005, Business Networks on the Island of Ireland, les entreprises interrogées identifiaient plusieurs faiblesses, qui, toutes, semblaient liées à un certain manque de volontarisme dans les politiques gouvernementales :

33. Department of Enterprise, Trade and Employment, Knowledge and Enterprise Clusters in Ireland: an Overview, op.cit., p. 15. "One of the major spillovers from the presence of major OEMs (note) in Ireland is a highly successful software sector."

34. South West Regional Authority, AT Cluster Benchmarking Report, June 2009, p. 19.

35. Aida Vadasi, "SME Networking Models and Examples from Northern Italy, Denmark and the UK", $5^{\text {th }}$ international conference on Management, Enterprise and Benchmarking, Budapest, June 1, 2007, p. 129. 
manque de fonds, manque de services (il était demandé un directory et un site web dédié), manque d'information auprès des entreprises irlandaises (petites et moyennes en particulier) sur l'existence des clusters et des networks, soutien insuffisant à l'internationalisation ${ }^{36}$. Cependant, les entreprises interrogées n'en soulignaient pas moins leur attachement à l'indépendance et à l'autonomie des structures : "Il est important que les réseaux soient gérés par les réseaux eux-mêmes et non par les agences ${ }^{37}$."

L'une des difficultés majeures rencontrées dans le fonctionnement des clusters et des networks en Irlande semblait donc être celle de concilier tous les intérêts, publics et privés, nationaux et régionaux, impliquant que l'intérêt premier des entreprises concernées n'était pas toujours respecté, comme l'ont noté Ash Amin et John Tomaney, en particulier dans le cas des clusters qui entraient dans une logique de développement régional :

Bien qu'ils aient souvent des effets bénéfiques pour les entreprises, les clusters ne sont pas toujours construits à cette fin et sont plus souvent motivés par des objectifs plus étendus ayant trait au développement économique régional et national dans l'intérêt public au sens plus large $\mathrm{e}^{38}$.

La crainte de voir l'objectif de développement régional devenir prioritaire sur l'intérêt premier des entreprises parties prenantes des clusters pouvait, en effet, ne pas être dénuée de fondement si l'on examine un document publié par Enterprise Ireland en 2003, Driving Regional Enterprise :

La croissance des clusters d'entreprises semblables est un aspect important pour le développement régional et un de ceux auxquels Enterprise Ireland est très attaché ${ }^{39}$.

Les craintes exprimées par les entreprises semblent cependant avoir été entendues. Les programmes de soutien à la construction de réseaux d'entreprises mis en œuvre depuis lors, comme Industry Led Research Networks Programme ${ }^{40}$ lancé par Enterprise Ireland, affichent désormais plus clairement la place laissée à l'initiative entrepreneuriale.

36. Maire Hunt, Gerald. Doyle, Dermott McDermott et Peter McCormack, op. cit., p. 52-53.

37. Ibid., p. 51. "It is important that networks are run by the networks themselves and not the agencies."

38. A. Amin, J. Tomaney, Behind the Myth of European Union: Prospects for Cohesion, op. cit., p. 310 : « Though they often result in gain for companies, clusters are not always established with that end in mind and are more often motivated by broader goals to do with regional and national economic development for the greater public good. "

39. Enterprise Ireland, Annual Report Accounts, 2003, chapter 4, p. 51: "The growth of clusters of similar businesses is a very important aspect to regional development and one to which Enterprise Ireland is fully committed."

40. Enterprise Ireland, Industry Led Research Networks Programme, [http://www.enterprise-ireland.com/en/ Research-Innovation/Companies/Collaborate-with-companies-research-institutes/Industry-Led-Research-NetworksProgramme.html], page consultée le 16 juin 2014. 
L'Irlande du Tigre celtique a donc su profiter de la dynamique des clusters et des networks développée depuis la fin des années 1980. Cette voie, en dépit de la crise économique, ou peut-être en raison même de celle-ci, reste très prometteuse, comme le rappelait, par exemple, le document Best Connected : Software from Ireland. A Strategy for Development of Indigenous Software Industry 2009-2013, publié par Enterprise Ireland en avril $2009^{41}$.

Lactualisation par Enterprise Ireland en $2011^{42}$ de l'étude sur les Business networks précédemment publiée en 2005 (voir supra) a clairement mis en évidence l'augmentation des clusters et des networks : 240 en 2011 contre 110 seulement en 2005.

Un certain nombre de lacunes ont été comblées, en particulier en matière de soutien public à ces structures, comme en a attesté, par exemple, un nouveau programme dédié aux programmes collaboratifs inter-entreprises de 2 milliards d'euros lancé en septembre 2012 par le Department of Jobs, Enterprise and Innovation irlandais ${ }^{43}$. Il importe cependant que l'aide apportée ne soit pas coercitive au point d'affaiblir la dynamique et l'autonomie entrepreneuriales qui sont au cœur même des modèles performants des clusters et des networks.

41. Enterprise Ireland, Best Connected: Software from Ireland. A Strategy for Development of Indigenous Software Industry 2009-2013, April 2009, [http://www.enterprise-ireland.com/NR/rdonlyres/117B1162-7B2D-41DC81DB-A4E6B8E48F82/0/SoftwarePublishedstrategy.pdf]. page consultée le 10 juin 2014.

42. Enterprise Ireland, Business networks on the island of Ireland, 2011.

43. Enterprise Ireland Press Releases, "New €2million clustering programme to promote cooperation between businesses and create more jobs: Creating clusters of strength is a key part of industrial strategy ", [http:// www.enterprise-ireland.com/en/news/pressreleases/2012-press-releases/new-\%E2\%82\%AC2million-clustering-programme-to-promote-cooperation-between-businesses-and-create-more-jobs-\%E2\%80\%93-minister-bruton.html], page consultée le 30 juin 2014. 